\title{
Con: Pure service delivery is no longer needed in global surgical missions
}

\author{
Faye M. Evans, MD • Mary T. Nabukenya, MBChB, MMed
}

Received: 17 October 2016/Accepted: 16 December 2016/Published online: 27 December 2016

(C) Canadian Anesthesiologists' Society 2016

Medical providers have a long history of volunteerism and service to those in need. For many of us, this concern for the health of others less fortunate represents why we were drawn to the medical profession in the first place. The Lancet Commission's Report on Global Surgery highlights the vast health inequalities that exist between high- and low-income countries. Five billion people do not have access to safe, affordable, surgical and anesthesia care when needed. Furthermore, 143 million additional surgical procedures are needed every year in low- and middle-income countries (LMICs) in order to save lives and prevent disability. ${ }^{1}$ Other important details are not as clearly defined. These relate to the most cost-effective, feasible, and replicable means to implement the systems needed to reduce these inequalities and promote health equity.

A topic often debated in the anesthesia and surgical global health community is the role of the "global surgical mission" as part of the solution. There are extremes of opinions-from those who think there is no role for global surgical missions to others who think the missions should have an important role. For many, the role depends on the nature and organization of the mission.

We contend that there is no role for pure service delivery in global surgical missions, except in cases where no other option exists. ${ }^{2}$ Surgical missions aim to address unmet healthcare needs in LMICs. While the surgical

F. M. Evans, MD ( $₫)$

Department of Anesthesiology, Perioperative, and Pain

Medicine, Boston Children's Hospital, 300 Longwood Avenue,

Bader 3, Boston, MA, USA

e-mail: Faye.Evans@childrens.harvard.edu

M. T. Nabukenya, MBChB, MMed

Department of Anaesthesia, Makerere University College of Health Sciences, Kampala, Uganda services and organizational structures they provide may differ, the real question is whether global surgical missions address unmet healthcare needs in an ethically responsible fashion. An additional question is whether there are evidence-based guidelines that can help to validate these practices.

There is little homogeneity in defining the term "global surgical mission". When Sykes performed a systematic literature review for empirical evidence of medical mission activities and outcomes during 1993-2013, the author reported 45 different terms used to describe short-term medical service trips. For purposes of his review, Sykes specifically described medical service trips (MSTs) as "trips in which volunteer medical providers from highincome countries (HICs) travel to LMICs to provide health care over one to eight weeks." 3 Even within these parameters, these service-based care projects can be delivered via a variety of existing service platforms. The most common type of mission is the "short-term" servicebased surgical trip of one to three weeks' duration. Typically organized around a specific surgical need (e.g., cleft lip/palate, pediatric cardiac surgery, or burn reconstruction), the primary goal of these efforts is to relieve the burden of disease at particular sites or in particular populations. These missions usually consist of self-contained teams comprising foreign surgeons, anesthesiologists, nurses, and support staff who travel together to pre-identified sites. These groups vary in their ability to adapt to host facilities and equipment. Some teams bring all of their equipment and supplies-the "surgical brigade model"-while others rely on host supplies or some combination of the two.

In contrast, there are longer-term platforms for global surgical missions that consist of self-contained surgical teams. These missions are led by non-governmental 
organizations such as Mercy Ships ${ }^{\mathrm{A}}$ or even faith-based specialty surgical hospitals. ${ }^{\mathrm{B}}$ These longer-term mission groups remain in a country for months to years, but they still carry their infrastructure with them and may or may not leave any physical structure behind. Shrime et al. reviewed articles relating to these various platforms to determine the effectiveness of each in delivering medical assistance in LMICs. They report that the "self-contained temporary platforms and specialized surgical centers appear to provide more effective and cost-effective care than the short-term surgical mission trips, except when no other delivery platform exists." What distinguishes these longer-term platforms from the short-term mission trip is that these ventures, in addition to providing clinical services, focus much of their efforts on finding ways to improve the healthcare systems of the country in which they are working.

Global surgical missions are still the most popular form of global outreach, but are they the most effective? Do they interfere with the local healthcare system by fostering a culture of dependence? In 2012, Martiniuk et al. reviewed articles evaluating the advantages and disadvantages of medical missions. In addition to describing the demographics surrounding these types of activities, they attempted to tabulate the perceived benefits and common criticisms. The authors' review revealed the paucity of literature providing quantitative data on costs, quality, regulation, and outcome. ${ }^{5}$ More recently, Sykes reviewed articles about MSTs in an effort to evaluate specific indicators, including treatment interventions, cost effectiveness, quality assessment, factors limiting MSTs from performing evaluations, reasons healthcare providers participate, role of education, and how those affected by MSTs perceive the visiting teams and their activities. Most of the author's questions were left unanswered, so he concluded that "reporting has dealt largely with outputs rather than outcomes". Sykes went on to explain that this criterion "falls short of the measures used to identify highquality evidence-based medicine". Indeed, the only outcomes reported were immediate post-surgical outcomes or other aspects of the mission, with little reported on the long-term effect of the treatment. ${ }^{3}$

Unfortunately, the data to support or condemn surgical missions are lacking. When considering the Lancet Commission's recommendation that surgical services be implemented into vertical national health strategies, it seems obvious that the traditional short-term "service" mission is really only a short-term solution. From this, a number of questions logically arise. Rather than abandon short-term surgical missions, should we focus our efforts

\footnotetext{
A https://www.mercyships.org.

B kijabehospital.org; https://cure.org/hospitals/.
}

on their improvement? Can they be structured such that they become components of a high-quality sustainable plan? Is it possible to transfer knowledge and expand skills to the local health system? Is it possible to incorporate short-term visits from surgical teams into longitudinal community-based health-care activities? There is a need for more cost-benefit analyses that not only measure clinical effectiveness but also evaluate skills transfer and quality improvements resulting from these mission trips.

Primum non nocere, "first do no harm". While the desire to help others is altruistic in nature, MSTs are complex undertakings with many opportunities for good intentions to go awry. There are those who think that humanitarian missions may actually cause more harm than good. ${ }^{6}$ Grimes et al. proposed a set of recommendations to help partnerships and missions maximize their effectiveness and minimize the risk of potential harm. These recommendations emphasize the following policies: 1) Individuals should use existing programs to identify appropriate placements that match their skill set. 2) Trips should be mindful of local needs and considerate of costs to the host institutions. 3) Priority should be given to training local healthcare providers. 4) Outcomes - not only clinical but also quality of life-should be monitored. 5) Regular trips to the same institution go a long way in building capacity and sustainability. ${ }^{7}$ If these types of guidelines are adopted by the international surgical and anesthesia community, they could help avoid some of the abovementioned mistakes and help promote sustainable change. Organizations participating in these types of activities need to make a commitment to record outcomes, not just for internal reporting but also for wider dissemination. An emphasis on evaluation rather than on the ideology that "any care is better than no care" will help improve the planning, implementation, and measurement of activity.

Surgical missions can be a part of the solution, but they need to be carefully integrated into the local healthcare system. ${ }^{1,8}$ There is no role for a pure service model in this solution, except in extreme circumstances when no anesthesia or surgical provider is available. There is always a community that can be engaged and supported in their efforts towards providing better health care.

\section{Contre : L'offre de services pure n'est plus nécessaire dans les missions chirurgicales internationales}

Le volontariat et le service aux démunis sont depuis longtemps inscrits dans l'histoire des fournisseurs de 
services médicaux. Pour bon nombre d'entre nous, cette préoccupation pour la santé des autres personnes moins bien nanties constitue la raison première pour laquelle nous nous sommes orientés vers le métier de médecin. Le Rapport de la Commission de la chirurgie mondiale de la revue The Lancet (Lancet Commission's Report on Global Surgery) souligne les inégalités criantes en matière de santé qui existent entre les pays à revenu faible et ceux à revenu élevé. Cinq milliards de personnes n'ont pas accès à des soins chirurgicaux et anesthésiques sécuritaires et abordables quand elles en ont besoin. En outre, il faudrait chaque année 143 millions d'interventions chirurgicales supplémentaires dans les pays à revenu faible à intermédiaire afin de sauver des vies et de prévenir l'invalidité. ${ }^{1}$ D'autres détails importants ne sont pas aussi bien définis. Par exemple, nous ne connaissons pas précisément les moyens les plus rentables, faisables et reproductibles afin de mettre en auvre les systèmes nécessaires à réduire ces inégalités et promouvoir l'équité en santé.

Un sujet souvent débattu dans la communauté internationale de l'anesthésie et de la chirurgie est le rôle de la «mission chirurgicale internationale » en tant que partie de la solution. Les opinions sont très partagées allant de ceux qui pensent que les missions chirurgicales internationales n'ont aucun rôle à jouer, à ceux qui estiment que les missions devraient tenir un rôle important. Pour un grand nombre, le rôle à assumer dépend de la nature et de l'organisation de la mission.

Selon nous, l'offre de services pure ne devrait plus jouer aucun rôle dans les missions chirurgicales internationales, sauf dans les cas où il n'existe pas d'autre option. ${ }^{2}$ Les missions chirurgicales ont pour objectif de répondre à des besoins de santé non satisfaits dans les pays à revenu faible à intermédiaire. Alors que les services chirurgicaux et les structures organisationnelles que ces missions offrent sont de nature diverse, la véritable question est de savoir si les missions chirurgicales internationales répondent aux besoins de soins de santé non satisfaits d'une façon responsable d'un point de vue déontologique. Une autre question est de savoir s'il existe des directives factuelles qui permettent de valider ces pratiques.

$\mathrm{La}$ définition du terme «mission chirurgicale internationale » est très peu homogène. Lorsque Sykes a réalisé une revue de littérature méthodique des données empiriques tirées des activités de missions médicales et de leurs résultats au cours de la période 1993-2013, il a rapporté pas moins de 45 différents termes utilisés pour décrire les voyages de services médicaux de courte durée. Aux fins de ce compte rendu, Sykes a spécifiquement décrit les voyages de services médicaux comme « des voyages dans lesquels des fournisseurs de soins médicaux bénévoles provenant de pays à revenu élevé se rendent dans des pays à revenu faible à intermédiaire pour offrir des soins de santé sur une période allant de une à huit semaines. ${ }^{3}$ Même si l'on se limite à ces paramètres, ces projets de soins fondés sur des services peuvent être offerts par différentes plateformes de service existantes. Le type de mission le plus répandu est le voyage chirurgical fondé sur les services « de courte durée », soit d'une durée allant de une à trois semaines. Ce type de mission est en général organisé pour répondre à un besoin chirurgical spécifique (par ex. chirurgie de bec-de-lièvre / fente palatine, chirurgie cardiaque pédiatrique, ou reconstruction après brûlure), et l'objectif principal de ces efforts est de soulager le fardeau de la maladie dans des régions ou chez des populations spécifiques. Ces missions consistent en général d'équipes autonomes composées de chirurgiens, d'anesthésiologistes, d'infirmières et de personnel de soutien provenant de l'étranger et voyageant ensemble vers des sites identifiés au préalable. Ces groupes varient dans leur capacité à s'adapter aux installations et au matériel dans le pays ou la région hôte. Certaines équipes apportent tout leur matériel et leurs fournitures : on parle alors d'un «modèle de brigade chirurgicale »; d'autres, en revanche, utilisent les fournitures sur place ou fonctionnent avec une combinaison des deux.

En revanche, il existe aussi des plateformes de missions chirurgicales internationales à plus long terme composées d'équipes chirurgicales autonomes. Ces missions sont souvent sous l'égide d'organismes non gouvernementaux tels que les Navires de l'espoir, ${ }^{\mathrm{A}}$ voire d'hôpitaux chirurgicaux spécialisés à vocation confessionnelle. ${ }^{\mathrm{B}}$ Ces groupes de missions à plus long terme demeurent dans un pays pendant des mois, voire des années, mais ils voyagent tout de même avec leurs infrastructures et laissent ou non une structure physique lorsqu'ils repartent. Shrime et coll. ont passé en revue les articles portant sur ces diverses plateformes afin de déterminer l'efficacité de chacune pour offrir une assistance médicale dans les pays à revenu faible à intermédiaire. Selon eux, «les plateformes temporaires autonomes et les centres chirurgicaux spécialisés semblent offrir des soins plus efficaces et rentables que les missions chirurgicales à court terme, sauf lorsqu'il n'existe pas d'autre plateforme de service. ${ }^{4} \mathrm{Ce}$ qui distingue ces plateformes à plus long terme des missions à court terme est le fait que ces missions, en plus de fournir des soins cliniques, concentrent plus d'efforts sur la recherche de façons d'améliorer les systèmes de santé du pays dans lequel elles opèrent.

Les missions chirurgicales internationales sont toujours la forme la plus populaire de rayonnement international, mais sont-elles vraiment les plus efficaces? N'interfèrent-elles

\footnotetext{
A https://www.mercyships.org.

B kijabehospital.org; https://cure.org/hospitals/.
} 
pas avec le système de santé local en favorisant une culture de la dépendance? En 2012, Martiniuk et coll. ont passé en revue des articles évaluant les avantages et les inconvénients des missions médicales. En plus de décrire les données démographiques entourant ce type d'activités, les auteurs ont tenté de tabuler les avantages perçus et les critiques récurrentes. Le compte rendu des auteurs a révélé le manque de littérature présentant des données quantitatives concernant les coûts, la qualité, la réglementation et les résultats de ces missions. ${ }^{5}$ Plus récemment, Sykes a passé en revue des articles concernant les voyages de services médicaux afin d'évaluer des indicateurs spécifiques, notamment les interventions thérapeutiques, la rentabilité, l'évaluation de la qualité, les facteurs empêchant ces missions de procéder à des évaluations, les raisons motivant les fournisseurs de soins de santé à y participer, le rôle de la formation, et comment les personnes affectées par ces missions perçoivent les équipes en visite et leurs activités. La plupart des questions de l'auteur sont restées sans réponse, c'est pourquoi il a conclu que « les comptes rendus portent principalement sur le rendement (soit le nombre de 'cas' traités ou d'interventions) plutôt que sur les résultats ». Sykes continue en expliquant que ce critère « n'est pas à la hauteur des mesures utilisées pour identifier une médecine factuelle de qualité élevée ». En effet, les seuls résultats rapportés étaient les pronostics post-chirurgicaux immédiats ou d'autres aspects de la mission, et très peu de données s'intéressaient à l'effet à long terme du traitement. ${ }^{3}$

Malheureusement, nous ne disposons pas de données appuyant ou condamnant les missions chirurgicales. Lorsqu'on considère la recommandation de la Commission de The Lancet, selon laquelle les services chirurgicaux devraient être mis en œuvre dans le cadre de stratégies verticales de santé nationale, il semble évident que la mission de « service » traditionnelle de courte durée ne constitue en fait qu'une solution à court terme. Il est donc logique que plusieurs questions soient soulevées. Plutôt que d'abandonner les missions chirurgicales de courte durée, devrions-nous concentrer nos efforts sur leur amélioration? Est-il possible de les structurer de façon à ce qu'elles s'inscrivent dans le cadre d'un plan durable de qualité élevée? Peut-on transférer nos connaissances et étendre nos compétences au système de santé local? Est-il possible d'intégrer les visites à court terme d'équipes chirurgicales à des activités de santé longitudinales et ancrées dans la communauté? Nous avons besoin d'analyses de coûts-avantages supplémentaires afin de mesurer non seulement l'efficacité clinique, mais également d'évaluer la réussite du transfert des compétences et les améliorations de la qualité résultant de ces missions.

Primum non nocere, « avant tout, ne pas nuire ». Bien que le désir d'aider les autres soit intrinsèquement altruiste, les voyages de services médicaux sont des entreprises complexes aux parcours semés d'embûches et ce, malgré toutes les bonnes intentions du monde. Certains sont d'avis que les missions humanitaires font en fait plus de mal que de bien. ${ }^{6}$ Grimes et coll. ont présenté un ensemble de recommandations afin d'aider les partenariats et les missions à optimiser leur efficacité et minimiser le risque de tort potentiel. Ces recommandations mettent l'accent sur les politiques suivantes: 1) les individus souhaitant participer à une mission devraient utiliser des programmes existants afin d'identifier des placements adaptés qui correspondent à leurs compétences; 2) les voyages doivent porter attention aux besoins locaux et faire attention aux coûts encourus par les institutions hôtes; 3) la priorité doit être la formation des fournisseurs de soins de santé locaux; 4) les résultats - non seulement cliniques mais également la qualité de vie - doivent être surveillés; 5) les voyages récurrents dans la même institution sont une excellente façon de renforcer les capacités et la durabilité. ${ }^{7}$ Si ce type de directives était adopté par la communauté de chirurgie et d'anesthésie internationale, elles pourraient contribuer à éviter certaines des erreurs mentionnées plus haut et aider à promouvoir un changement durable. Les organisations qui participent à ce type d'activités doivent s'engager à documenter leurs résultats, non seulement à des fins de communication interne, mais également pour une diffusion plus large. Une emphase sur l'évaluation plutôt que sur l'idéologie selon laquelle « des soins, quels qu'ils soient, sont mieux que pas de soins » permettra d'améliorer la planification, la mise en œuvre et l'évaluation de ces activités.

Les missions chirurgicales peuvent faire partie de la solution, mais il faut qu'elles soient intégrées avec soin dans le système de soins de santé local. ${ }^{1,8}$ Dans cette solution, un modèle de services pur ne joue plus aucun rôle, sauf dans les circonstances extrêmes où il n'y a pas de fournisseur d'anesthésie ou de chirurgie. Il existe toujours une communauté qui peut s'impliquer et qu'on peut soutenir dans ses efforts pour offrir de meilleurs soins de santé.

Conflicts of interest None declared.

Editorial responsibility This submission was handled by Dr. Hilary P. Grocott, Editor-in-Chief, Canadian Journal of Anesthesia.

\section{Conflit d'intérêt Aucun.}

Responsabilité éditoriale Cet article a été traité par Dr Hilary P. Grocott, rédacteur en chef, Journal canadien d'anesthésie.

\section{References}

1. Meara JG, Leather AJ, Hagander L, et al. Global Surgery 2030: evidence and solutions for achieving health, welfare, and economic development. Lancet 2015; 386: 569-624. 
2. Werner D, Maxwell J, Thuman C, Where There is No Doctor: A Village Health Care Handbook. Macmillan Education Limited; 1993.

3. Sykes KJ. Short-term medical service trips: a systematic review of the evidence. Am J Public Health 2014; 104: e38-48.

4. Shrime MG, Sleemi A, Ravilla TD. Charitable platforms in global surgery: a systematic review of their effectiveness, costeffectiveness, sustainability, and role training. World J Surg 2015; 39: 10-20.

5. Martiniuk AL, Manouchehrian M, Negin JA, Zwi AB. Brain gains: a literature review of medical missions to low and middle-income countries. BMC Health Serv Res 2012; 12: 134.
6. Welling DR, Ryan JM, Burris DG, Rich NM. Seven sins of humanitarian medicine. World J Surg 2010; 34: 466-70.

7. Grimes CE, Maraka J, Kingsnorth AN, Darko R, Samkange CA, Lane $R H$. Guidelines for surgeons on establishing projects in lowincome countries. World J Surg 2013; 37: 1203-7.

8. Farmer PE, Kim JY. Surgery and global health: a view from beyond the OR. World J Surg 2008; 32: 533-6. 